Article

\title{
Novel Strategies Using Total Gastrodin and Gastrodigenin, or Total Gastrodigenin for Quality Control of Gastrodia elata
}

\author{
Chunlan Tang *, Bingchu Wu, Jinyi Wu, Zheng Zhang and Bocheng Yu \\ Department of Preventative Medicine, Medical School of Ningbo University, Ningbo 315211, China; \\ bingchu97@163.com (B.W.); wujiny@foxmail.com (J.W.); ZZ1171987191@163.com (Z.Z.); \\ a13757451117@163.com (B.Y.) \\ * Correspondence: chunlant@163.com; Tel.: +86-574-8760-9894
}

Received: 29 December 2017; Accepted: 27 January 2018; Published: 29 January 2018

\begin{abstract}
Gastrodia elata Blume (G. elata), a traditional Chinese medicine, is widely used for treatment of various neuro dysfunctions. However, its quality control is still limited to the determination of gastrodin. In the present study, two novel strategies based on quantitative evaluation of total gastrodin and gastrodigenin with base hydrolysis and total gastrodigenin with base-enzymatic hydrolysis followed by HPLC-FLD were put forward and successfully applied to evaluate the quality of 47 batches of G. elata from eight localities. Meanwhile, a systematic comparison of the novel strategy with the multiple markers and the Pharmacopeia method was performed. The results showed that the parishins category could be completely hydrolyzed to gastrodin by sodium hydroxide solution, and gastrodin could further utterly hydrolyze to gastrodigenin with $\beta$-D-glucosidase buffer solution. The contents of total gastrodin and gastrodigenin ranged from $1.311 \%$ to $2.034 \%$, and total gastrodigenin from $0.748 \%$ to $1.120 \%$ at the eight localities. From the comparison, we can conclude that the two novel strategies can comprehensively reveal the characteristics of overall active ingredients in G. elata for quality control. The present study provides a feasible and credible strategy for the quality control of G. elata, suggesting a revision of the latest Chinese Pharmacopoeia or European Pharmacopoeia methods for the modernization of G. elata use.
\end{abstract}

Keywords: total gastrodin; total gastrodigenin; base-enzymatic hydrolysis; HPLC-FLD

\section{Introduction}

Gastrodia elata Blume (G. elata), the tuber of the orchid, is a traditional Chinese medicine (TCM) extensively used against convulsions, vertigo, paralysis, epilepsy, tetanus, asthma and immune dysfunctions [1-6]. Gastrodin (GAS) is widely considered as the main and bioactive ingredient in G. elata for its sedative, anticonvulsive and neuroprotective effects [7-10]. Therefore, a large number of studies on the pharmacokinetics, and pharmacological studies of GAS have been carried out [11-13]. In general, TCMs contain a large number of ingredients, many of which exert favorable pharmacological activity [14,15]. Therefore, choosing only one or two ingredients as markers for quality control of TCM is not accord with the holistic characteristics of TCMs.

With the development of phytochemistry, a lot of active ingredients, including gastrodigenin ( $p$-hydroxybenzyl alcohol, HBA), the parishins, nucleosides, etc. were identified in G. elata [16,17], and these ingredients revealed preferable efficacy $[18,19]$, particularly in terms of neuroprotective efficacy [20-23]. Therefore, the use of GAS as the single marker is not enough for the quality control of G. elata. In the Chinese Pharmacopoeia (2015 version), HBA was added as another marker, that is, the GAS and HBA both control the quality of G. elata. In addition, $\mathrm{Li}$ et al. reported the use of 5-hydroxy-methyl-2-furaldehyde, parishin B (PB) and parishin C (PC) as markers of G. elata by 
fingerprint-efficacy relationship modelling [24]. Ma et al. employed GAS, HBA, parishin E (PE), PB, PC and parishin (PA) as markers to evaluate the quality of G. elata. However, as reported, G. elata contains almost eighty ingredients [25]. Among them, some exert good and various pharmacological activity, so only choosing GAS, HBA or PC as markers is not enough to cover most ingredients and explain the overall characteristic of G. elata, such as its pharmacokinetic or pharmacodynamic properties [26]. Therefore, some researchers develop the use of fingerprint methods or multi-component patterns for the quality control and origin discrimination of G. elata by similarity index [27-29]. Nevertheless, fingerprint evaluation of TCM quality of still has many challenges, including how to definite the common peaks, how to solve the problems of background drift, overlapping peaks, noisy peaks, etc.

The present study aimed to investigate two novel strategies for quality control of G. elata, which could comprehensively reveal the characteristics of overall active ingredients in G. elata, and could be conveniently and effectively adapted in various laboratory and clinical applications. Therefore, two novel strategies, based on quantitative evaluation of total GAS and HBA with base hydrolysis (TGH-B), and total HBA with base-enzymatic hydrolysis (TH-BE) followed by HPLC-FLD techniques, were carried out and applied to evaluate 47 batches of $G$. elata from eight localities. In addition, the comparison of the proposed strategies with multiple markers and the Pharmacopeia method for quality control of G. elata was also investigated [30,31]. The success of the novel strategy suggest a revision of the Pharmacopoeia method and lays the foundation for the modernization of G. elata use.

\section{Results}

\subsection{Development of the Method}

Our previous studies have reported the fluorescence properties of the main phenols in G. elata [32]. Considering the different instrumentation, the excitation and emission wavelength were further optimized by scanning the fluorescence spectrum of each compound. The results showed that the maximum excitation and emission wavelengths were at $275 \mathrm{~nm}$ and $295 \mathrm{~nm}$, respectively. In addition, the flow rate, column temperature and gradient elution condition were optimized to achieve well separated and sharp peaks. After optimization of the chromatographic conditions, six compounds, including GAS, HBA, PE, PB, PC and PA (Figure 1), were effectively separated in 15 min when the column was eluted in a gradient of methanol and water containing $0.5 \%$ formic acid. The retention times of GAS, HBA, PE, PB, PC and PA were 6.64, 8.27, 9.17, 10.07, 10.53, $11.20 \mathrm{~min}$, respectively (Figure 2A).

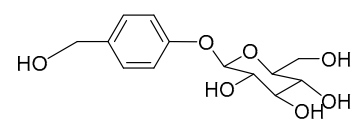

Gastrodin (GAS)

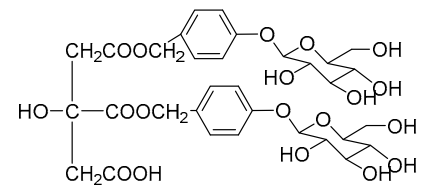

Parishin B (PB)

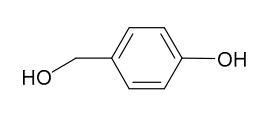

Gastrodigenin (HBA)

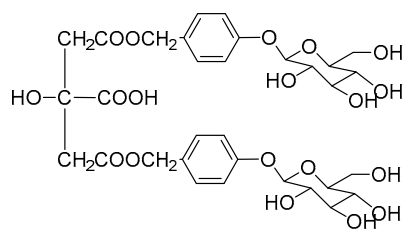

Parishin C (PC)

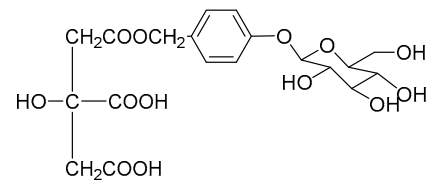

Parishin E (PE)

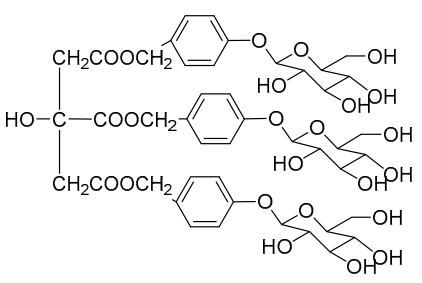

Parishin (PA)

Figure 1. The structures of gastrodin (GAS), gastrodigenin (HBA), parishin $E(P E)$, parishin $B(P B)$, parishin C (PC) and parishin (PA). 

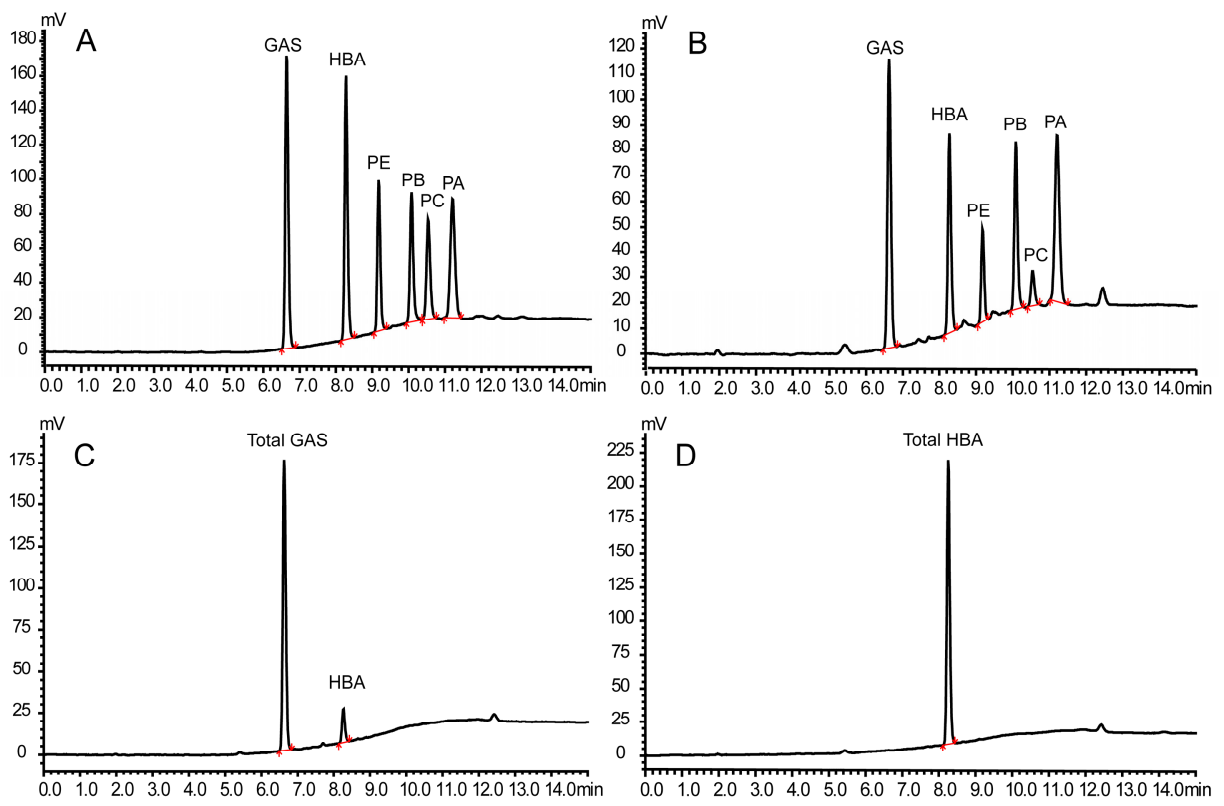

Figure 2. Representative chromatograms: (A) standard solution including GAS, HBA, PE, PB, PC and PA; (B) extract of G. elata; (C) extract of G. elata after base hydrolysis; (D) extract of G. elata after base-enzymatic hydrolysis. The injected volume of extract of G. elata was $10 \mu \mathrm{L}$, and the hydrolyzed samples was $2 \mu \mathrm{L}$.

The regression curve presented high linearity, with a coefficient $\left(r^{2}\right)$ larger than 0.99 within the concentration range from 0.05 to $10 \mu \mathrm{g} / \mathrm{mL}$, demonstrating its suitability for quantitative analysis of GAS, HBA, PE, PB, PC, PA. The limit of quantitation (LOQ) for six compounds was $0.05 \mu \mathrm{g} / \mathrm{mL}$. The RSD and RE at three concentration levels (five replicates) of GAS, HBA, PE, PB, PC, PA were all within $\pm 15 \%$, indicating the good precision and trueness.

The above results are summarized in Table 1 . In addition, the extraction recoveries and matrix effects of GAS, HBA, PE, PB, PC and PA were $95.28 \%, 92.20 \%, 110.27 \%, 114.98 \%, 99.52 \%, 101.32 \%$ and $94.48 \%, 97.63 \%, 101.93 \%, 100.79 \%, 106.79 \%, 103.84 \%$, respectively (Table 2). These results indicated that the present method was reliable and reproducible for the quantitative analysis of GAS, HBA, PE, $\mathrm{PB}, \mathrm{PC}$ and PA.

Table 1. Intra- and inter-day precision and trueness, linearity and limit of quantitation (LOQ) of gastrodin (GAS), gastrodigenin (HBA), parishin E (PE), parishin B (PB), parishin C (PC) and parishin (PA).

\begin{tabular}{|c|c|c|c|c|c|c|c|c|c|}
\hline \multirow{3}{*}{ Compounds } & \multicolumn{7}{|c|}{ Precision and Trueness $(n=5)$} & \multirow{3}{*}{ Linearity } & \multirow{3}{*}{ LOQ } \\
\hline & \multirow{2}{*}{$\begin{array}{l}\text { Concentration } \\
(\mu \mathrm{g} / \mathrm{mL})\end{array}$} & \multicolumn{3}{|c|}{ Intra-Day } & \multicolumn{3}{|c|}{ Inter-Day } & & \\
\hline & & Mean \pm SD & RSD (\%) & RE (\%) & Mean \pm SD & RSD (\%) & RE (\%) & & \\
\hline \multirow{3}{*}{ GAS } & 0.2 & $0.19 \pm 0$ & 0.94 & -5.05 & $0.18 \pm 0$ & 3.37 & -6.67 & \multirow{3}{*}{$\begin{array}{c}y=9.12 \times 10^{5} x+ \\
\quad 6.69 \times 10^{4}\end{array}$} & \multirow{3}{*}{0.5} \\
\hline & 1.2 & $1.18 \pm 0$ & 0.20 & -1.70 & $1.16 \pm 0.02$ & 1.85 & -3.50 & & \\
\hline & 6 & $5.97 \pm 0.02$ & 0.36 & -0.46 & $5.86 \pm 0.11$ & 1.89 & -2.37 & & \\
\hline \multirow{3}{*}{ HBA } & 0.2 & $0.22 \pm 0$ & 0.20 & 9.58 & $0.21 \pm 0$ & 2.27 & 6.55 & \multirow{3}{*}{$\begin{aligned} y= & 2.07 \times 10^{6} x- \\
& 7.44 \times 10^{4}\end{aligned}$} & \multirow{3}{*}{0.5} \\
\hline & 1.2 & $1.19 \pm 0$ & 0.29 & -0.48 & $1.16 \pm 0.03$ & 2.41 & -3.08 & & \\
\hline & 6 & $5.97 \pm 0.01$ & 0.18 & -0.47 & $5.75 \pm 0.14$ & 2.42 & -4.10 & & \\
\hline \multirow{3}{*}{ PE } & 0.2 & $0.17 \pm 0$ & 3.55 & -14.97 & $0.17 \pm 0$ & 3.00 & -14.77 & \multirow{3}{*}{$\begin{aligned} y= & 1.65 \times 10^{5} x- \\
6.01 & \times 10^{3}\end{aligned}$} & \multirow{3}{*}{0.5} \\
\hline & 1.2 & $1.10 \pm 0.02$ & 1.41 & -8.64 & $1.06 \pm 0.03$ & 2.95 & -12.02 & & \\
\hline & 6 & $5.60 \pm 0.03$ & 0.46 & -6.73 & $5.45 \pm 0.11$ & 2.10 & -9.11 & & \\
\hline \multirow{3}{*}{ PB } & 0.2 & $0.17 \pm 0$ & 5.10 & -14.16 & $0.17 \pm 0$ & 2.33 & -13.19 & \multirow{3}{*}{$\begin{array}{c}y=2.16 \times 10^{5} x+ \\
5.31 \times 10^{3}\end{array}$} & \multirow{3}{*}{0.5} \\
\hline & 1.2 & $1.09 \pm 0.02$ & 1.91 & -9.10 & $1.07 \pm 0.02$ & 2.31 & -10.54 & & \\
\hline & 6 & $5.71 \pm 0.04$ & 0.75 & -4.85 & $5.71 \pm 0.09$ & 1.56 & -4.83 & & \\
\hline \multirow{3}{*}{ PC } & 0.2 & $0.18 \pm 0.02$ & 8.52 & -9.00 & $0.21 \pm 0.02$ & 11.44 & 6.43 & \multirow{3}{*}{$\begin{array}{c}y=1.77 \times 10^{5} x+ \\
5.64 \times 10^{3}\end{array}$} & \multirow{3}{*}{0.5} \\
\hline & 1.2 & $1.12 \pm 0.02$ & 1.79 & -6.81 & $1.10 \pm 0.01$ & 1.00 & -8.57 & & \\
\hline & 6 & $5.77 \pm 0.03$ & 0.50 & -3.82 & $5.72 \pm 0.12$ & 0.02 & -4.65 & & \\
\hline \multirow{3}{*}{ PA } & 0.2 & $0.19 \pm 0$ & 5.11 & -4.88 & $0.17 \pm 0$ & 1.48 & -12.72 & \multirow{3}{*}{$\begin{array}{c}y=1.98 \times 10^{5} x+ \\
5.10 \times 10^{3}\end{array}$} & \multirow{3}{*}{0.5} \\
\hline & 1.2 & $1.11 \pm 0.02$ & 1.56 & -7.88 & $1.05 \pm 0.03$ & 3.36 & -12.36 & & \\
\hline & 6 & $5.38 \pm 0.01$ & 0.22 & -10.26 & $5.39 \pm 0.16$ & 3.01 & -10.24 & & \\
\hline
\end{tabular}


Table 2. The extraction recoveries and matrix effects of GAS, HBA, PE, PB, PC and PA.

\begin{tabular}{cccccc}
\hline Compound & Origin $(\mu \mathrm{g} / \mathrm{mL})$ & Spiked $(\mu \mathrm{g} / \mathrm{mL})$ & Determined $(\mu \mathrm{g} / \mathrm{mL})$ & Recovery $(\%)$ & Matrix Effect $(\%)$ \\
\hline GAS & 0.7021 & 0.5 & $1.1785 \pm 0.0108$ & $95.28 \pm 2.16$ & $94.88 \pm 0.02$ \\
HBA & 0.1361 & 0.5 & $0.5970 \pm 0.0199$ & $92.20 \pm 3.98$ & $97.63 \pm 0.62$ \\
PE & 1.2966 & 0.5 & $1.8479 \pm 0.0065$ & $110.27 \pm 1.29$ & $101.93 \pm 0.75$ \\
PB & 2.3254 & 0.5 & $2.9022 \pm 0.1082$ & $114.98 \pm 20.96$ & $100.79 \pm 0.17$ \\
PC & 0.6598 & 0.5 & $1.1573 \pm 0.0605$ & $99.52 \pm 12.11$ & $106.79 \pm 1.63$ \\
PA & 2.6140 & 0.5 & $3.1205 \pm 0.0574$ & $101.32 \pm 11.47$ & $103.84 \pm 1.07$ \\
\hline
\end{tabular}

\subsection{Quality Evaluation by Pharmacopoeia Indices}

GAS and HBA are used as quality guideline of G. elata in the Chinese Pharmacopoeia (2015 version), where the added content of GAS and HBA was set as not less than $0.25 \%$. GAS is used as quality criterion in the European Pharmacopoeia, where the content of GAS is set as not less than $0.20 \%$. Therefore, this study firstly determined the content of GAS and HBA of 47 batches of G. elata from eight localities using the developed method. The results showed that the added content of GAS and HBA at the eight localities ranged from $0.181 \%$ to $0.942 \%$, while GAS was $0.106 \%$ to $0.918 \%$, which almost meets the criteria of both pharmacopoeias, except for seven samples which were marked in red in Supplementary Material Table S1. The above results indicated that the quality of G. elata from various drugstores are almost compliant with the guidelines. In addition, the quality of G. elata from eight localities was sorted according to the average of content sum of GAS and HBA or the single GAS, the order was as follows: Anhui $>$ Xizang $>$ Zhejiang $>$ Sichuan $>$ Yunnan $>$ Guizhou $>$ Jilin $>$ Shanxi (Table 3).

Table 3. The content of GAS, HBA, PE, PB, PC, PA and the sum in extract of G. elata.

\begin{tabular}{ccccccccc}
\hline $\begin{array}{c}\text { Content } \\
(\%)\end{array}$ & GAS & HBA & $\begin{array}{c}\text { GAS + } \\
\text { HBA }\end{array}$ & PE & PB & PC & PA & $\begin{array}{c}\text { Sum of Six } \\
\text { Compounds }\end{array}$ \\
\hline Yunnan & $0.356 \pm 0.172$ & $0.093 \pm 0.056$ & 0.449 & $0.856 \pm 0.226$ & $0.725 \pm 0.204$ & $0.196 \pm 0.072$ & $1.475 \pm 0.448$ & 3.700 \\
Sichuan & $0.378 \pm 0.231$ & $0.097 \pm 0.062$ & 0.475 & $0.620 \pm 0.166$ & $0.540 \pm 0.158$ & $0.128 \pm 0.040$ & $0.930 \pm 0.455$ & 2.693 \\
Anhui & $0.485 \pm 0.174$ & $0.056 \pm 0.042$ & 0.541 & $0.583 \pm 0.076$ & $0.546 \pm 0.113$ & $0.131 \pm 0.034$ & $1.015 \pm 0.552$ & 2.815 \\
Zhejiang & $0.410 \pm 0.288$ & $0.070 \pm 0.030$ & 0.480 & $0.619 \pm 0.181$ & $0.541 \pm 0.150$ & $0.128 \pm 0.042$ & $1.269 \pm 0.701$ & 3.037 \\
Guizhou & $0.353 \pm 0.102$ & $0.044 \pm 0.016$ & 0.397 & $0.544 \pm 0.167$ & $0.439 \pm 0.164$ & $0.127 \pm 0.037$ & $0.768 \pm 0.338$ & 2.274 \\
Jilin & $0.283 \pm 0.170$ & $0.092 \pm 0.043$ & 0.375 & $0.832 \pm 0.143$ & $0.561 \pm 0.191$ & $0.134 \pm 0.049$ & $0.990 \pm 0.678$ & 2.891 \\
Shanxi & $0.279 \pm 0.040$ & $0.080 \pm 0.024$ & 0.359 & $0.614 \pm 0.076$ & $0.569 \pm 0.072$ & $0.148 \pm 0.014$ & $1.085 \pm 0.156$ & 2.774 \\
Xizang & $0.478 \pm 0.161$ & $0.062 \pm 0.030$ & 0.540 & $0.799 \pm 0.135$ & $0.681 \pm 0.043$ & $0.127 \pm 0.021$ & $1.256 \pm 0.252$ & 3.402 \\
\hline
\end{tabular}

Data, expressed as percentage of crude drug, were the mean \pm SD of different batches, and each batch at three experiments.

\subsection{Quality Evaluation by Multi-Markers}

The fluorescence fingerprint chromatograms of $G$. elata extract were produced and are depicted in Figure 2B. A total of six peaks which were assigned as GAS, HBA, PE, PB, PC and PA, respectively, were unequivocally identified by comparing their retention times with those of reference substances. In addition, the results showed that the six active compounds were all detected at all batches of G. elata from eight localities. The quantitative analysis results showed that the average content of PE, PB, PC and PA was highest at Yunnan region, GAS at Anhui region, and HBA at Sichuan region. In addition, the correlation analysis of locality and content of active compounds was carried out using the SIMCA 14.1 software (Umetrics, Umea, Sweden). As seen from Figure 3, it can be obtained that the four parishins and Yunnan region were almost at the same position. The feature distinguishing the Yunnan region from the other seven regions was the parishins. Shanxi, Sichuan and Jinlin can be classified into the same cluster and were close to the HBA, indicating that they all possess relative higher contents of HBA. Anhui, Zhejiang and Xizang could be placed into the same group, which possesses a relatively high content of GAS. In addition, Guizhou region is the furthest from the four parishins, showing the lowest content of these compounds. The total content of the six active compounds in G. elata from the eight localities ranged from $2.274 \%$ to $3.700 \%$ (Table 2). According to the total content, the quality 
of G. elata from the eight localities could be ranked as follows: Yunnan > Xizang > Zhejiang > Jilin > Anhui $>$ Shanxi $>$ Sichuan $>$ Guizhou.

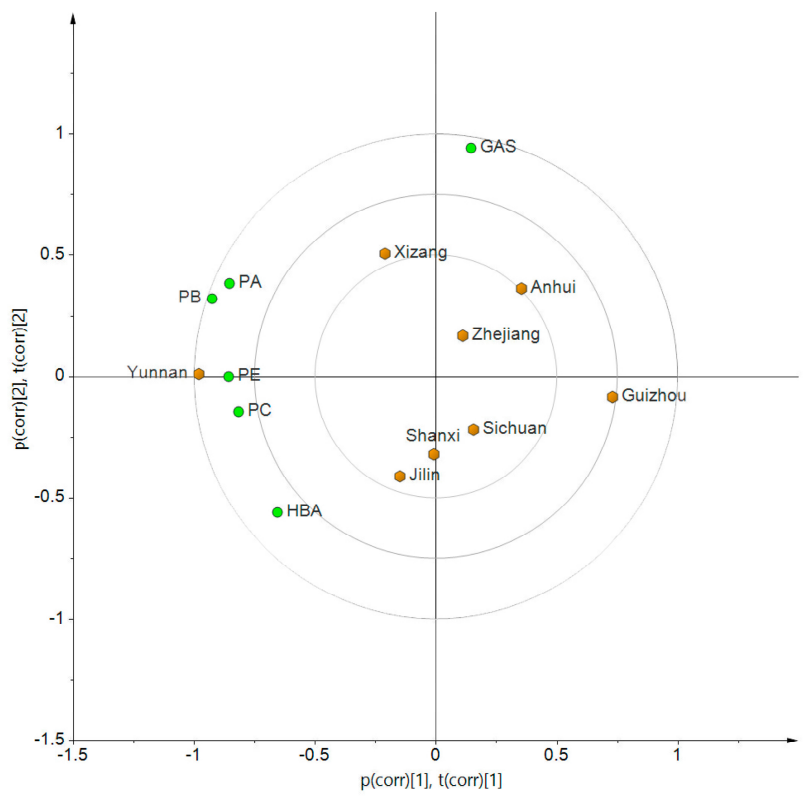

Figure 3. Biplot of the average content of GAS, HBA, PE, PB, PC and PA with eight localities of G. elata.

\subsection{TGH-B Strategy by Total GAS and HBA}

Our previous study showed that PA could hydrolyze to GAS [33], and Li et al. reported that there are over twenty parishins in G. elata [34]. In addition, considering the difficulty of preparation of the parishins and the instability of the parishins, this study adopted a hydrolysis experiment, whereby the parishins were hydrolyzed to GAS by an alkaline solution, and then the total GAS and HBA was determined after base hydrolysis for quality evaluation. The hydrolytic condition including temperature $\left(60-80^{\circ} \mathrm{C}\right)$, time $(0.5-2 \mathrm{~h})$ and alkali concentration $(0.5-3 \mathrm{~mol} / \mathrm{L})$ were optimized. The results showed that no remarkable variation was observed under the above-mentioned conditions. Therefore, the temperature of $80^{\circ} \mathrm{C}$, time of $0.5 \mathrm{~h}$ and alkali concentration of $0.5 \mathrm{~mol} / \mathrm{L}$ were chosen as hydrolytic conditions. Under these conditions, the samples were hydrolyzed and further analyzed. Comparing the chromatograms of hydrolysis before and after (Figure 2A,C), all peaks of parishins have disappeared and the peak of GAS was remarkably increased, indicating that the parishins were all completely hydrolyzed under the present conditions. Then the content of total GAS and HBA were determined, which were ranged from $1.311 \%$ to $2.034 \%$ for the eight localities. According to the content sum of total GAS and HBA, the quality of G. elata from the eight localities followed the order Xizang $>$ Zhejiang $>$ Yunnan $>$ Anhui $>$ Jilin $>$ Sichuan $>$ Shanxi $>$ Guizhou (Figure 4A).
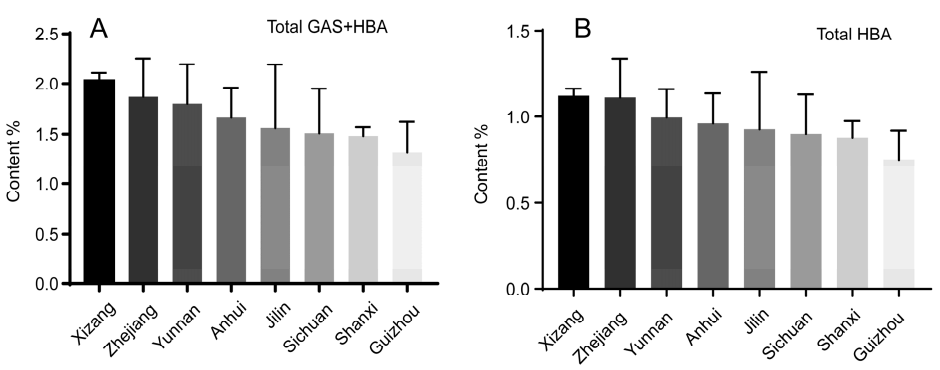

Figure 4. The content of components of G. elata after hydrolysis: (A) the sum of total GAS and HBA in G. elata after base hydrolysis; (B) total HBA in G. elata after base-enzymatic hydrolysis. Data, expressed as percentage of crude drug, were the mean \pm SD of different batches, and each batch at three experiments. 


\subsection{TG-BE Strategy by Total HBA}

Many studies have reported that GAS could metabolize to HBA, and HBA could cross the blood-brain barrier and exert its neuroprotective effects [35-37]. Therefore, this study hydrolyzed the parishins to produce GAS by alkaline solution, and further hydrolyzed GAS to HBA by enzyme solution, employing the total HBA level as a quality index. Firstly, the condition of enzymatic hydrolysis was investigated by L9 $\left(3^{4}\right)$ orthogonal experiment. The orthogonal experiment result showed that GAS could be totally hydrolyzed to HBA at an enzyme concentration of $200 \mathrm{U}$, rotation speed of $1000 \mathrm{rpm}$, and a time of $3 \mathrm{~h}$ (see Supplementary Material Table S2). Under these conditions, all batches of extracts of $G$. elata were hydrolyzed and then further analyzed using HPLC-FLD. From the fluorescence chromatogram (Figure 2D), it can be obtained that a single HBA peak was detected and the peak height was observably increased, suggesting that the parishins and GAS were all utterly decomposed to HBA. The content of total HBA ranged between $0.748 \%$ and $1.120 \%$ for the eight localities. According to the content of total HBA, we can rank the quality of G. elata from the eight localities in the following order: Xizang $>$ Zhejiang $>$ Yunnan $>$ Anhui $>$ Jilin $>$ Sichuan $>$ Shanxi $>$ Guizhou (Figure 4B).

\section{Discussion}

Many previous studies mainly used GAS as the marker of G. elata, and a large number of studies on the pharmacokinetics, and pharmacological studies of GAS were carried out [38-40]. With the development of phytochemistry, a lot of active compounds, including HBA, the parishins, the nucleosides, etc. were identified, so the use of GAS as the single marker was not enough for the quality control of G. elata. In the latest version of Chinese Pharmacopoeia in 2015, HBA was added as another marker, that is, the GAS and HBA both control the quality of G. elata, but as we all know, each TCM usually contains hundreds of ingredients. Among them, some exert good and varied pharmacological activity, so only choosing one or a few of the ingredients as markers is not enough to explain the overall characteristics of a TCM. Therefore, two novel TGH-B and TH-BE strategies using the sum of total GAS and HBA, and total HBA were proposed and successfully applied for quality evaluation of $G$. elata in 47 batches from eight localities. A comparison between the TGH-B and TH-BE strategies with the Pharmacopoeia method and multiple makers method was carried out. The results are shown in Table 4. We can observe that the results of determination of total GAS and HBA or total HBA were basically identical, which may be on account of two reasons. One is that GAS was thoroughly hydrolyzed to HBA by $\beta$-D-glucosidase enzymes. Another is that few isomers of GAS, which could be hydrolyzed to HBA by $\beta$-D-glucosidase, exist in G. elata, or the isomers of GAS could not convert into HBA by enzymatic hydrolysis. Therefore, the order of the content of total GAS and HBA, and total HBA was basically consistent for the eight localities. However, comparing the multiple markers method with the TGH-B and TH-BE strategies, a few turned out different. It has been reported that over 20 kinds of parishins such as parishin K, parishin N, etc. are found in G. elata [34], not only $\mathrm{PE}, \mathrm{PB}, \mathrm{PC}$ and PA. According to the structure of the parishins, these compounds may all hydrolyze to GAS, so if only four parishins are used, the content of other parishins is not considered, which resulted in the differences observed. Homoplastically, if only GAS and HBA, or only GAS are determined, many active ingredients are not considered, which could not represent the overall properties and result in a quite different order from the other three methods. The novel strategies by determination of total GAS and HBA, or total HBA after base or base-enzymatic hydrolysis, covered most active ingredients, which all possessed the active $p$-hydroxybenzyl alcohol structure and could be hydrolyzed by alkali or enzymatic solutions. Therefore, the two strategies can comprehensively reflect the content of total active ingredients in G. elata, which could provide better scientific strategies for the quality control of G. elata, and suggest that a revision of the latest Chinese or European pharmacopoeias for the modernization of G. elata use should be considered. 
Table 4. Comparison of four strategies for quality control of G. elata.

\begin{tabular}{ccl}
\hline Strategy & Marker & Order of Quality \\
\hline Pharmacopoeia & GAS and HBA or single GAS & Anhui $>$ Xizang $>$ Zhejiang $>$ Sichuan $>$ Yunnan $>$ Guizhou $>$ Jilin $>$ Shanxi \\
Multi-markers & GAS, HBA, PE, PB, PC and PA & Yunnan $>$ Xizang $>$ Zhejiang $>$ Jilin $>$ Anhui $>$ Shanxi $>$ Sichuan $>$ Guizhou \\
TGH-B & Total GAS, HBA & Xizang $>$ Zhejiang $>$ Yunnan $>$ Anhui $>$ Jilin $>$ Sichuan $>$ Shanxi $>$ Guizhou \\
TH-BE & Total HBA & Xizang $>$ Zhejiang $>$ Yunnan $>$ Anhui $>$ Jilin $>$ Sichuan $>$ Shanxi $>$ Guizhou \\
\hline
\end{tabular}

\section{Materials and Methods}

\subsection{Chemicals and Reagents}

Eight localities in China were selected and 3-10 batches of G. elata were purchased at each locality from various pharmacies (see Supplementary Material Table S3). Voucher specimens were deposited in the Key Laboratory of Pathological and Physiological Technology, Ningbo, Zhejiang. Parishin (PA > 98\%), parishin B (PB > 95\%), parishin C (PC > 95\%), and gastrodigenin (HBA > 98\%) was isolated and purified from the dried roots of G. elata and identified in our lab [15,25]. Gastrodin (GAS > 98\%) and parishin E (PE > 95\%) were purchased from Chengdu Biopurify Phytochemicals Co., Ltd. (Chengdu, China). $\beta$-D-Glucosidase was purchased from Wuhan Dahua Pharmaceutical Co., Ltd. (Wuhan, China). Methanol of chromatographic grade were obtained by Tedia (Fairfield, $\mathrm{OH}, \mathrm{USA}$ ). Formic acid of chromatographic grade, sodium hydroxide, sodium acetate and acetic acid of analytical grade were purchased from Aladdin (Shanghai, Chuina). Calibration solution of $\mathrm{pH} 4.1$ was purchased from Shanghai Solarbio Life Science Co., Ltd. (Shanghai, China). Hydrochloric acid of analytical grade was purchased from Zhejiang Zhongxing Chemical Reagent Co., Ltd. (Zhejiang, China). Ultrapure water was obtained by a water purification system (Milli integral 5, Millipore, Billerica, MA, USA).

\subsection{Instrumentation}

The chromatographic analysis was carried out on an HPLC-FLD system (Shimadzu, Kyoto, Japan), equipped with a pump, autosampler, degasser, column oven and fluorescence detector. Separation was performed on a Shimadzu reverse phase InertSustain C18 HPLC column $(4.6 \times 150 \mathrm{~mm}, 5 \mu \mathrm{m})$ with column oven $30{ }^{\circ} \mathrm{C}$. The elution gradient was composed of $0.5 \%$ formic acid aqueous solution (C) and methanol (A) at a flow rate of $1 \mathrm{~mL} / \mathrm{min}$. Optimized separation of targets was obtained according to a linear gradient that increased from $5 \%$ to $40 \% \mathrm{~A}$ in $5 \mathrm{~min}$ and held for additional $10 \mathrm{~min}$. The injection volume was set at $10 \mu \mathrm{L}$ for extracted sample and $2 \mu \mathrm{L}$ for hydrolytic sample. The excitation and emission wavelengths of the fluorescence analysis were set at $275 \mathrm{~nm}$ and $295 \mathrm{~nm}$, respectively.

\subsection{Preparation of Standard Solution and Extract of G. elata}

PA, PB, PC, PE, GAS and HBA powders were separately dissolved in 50\% methanol-water at a final concentration of $1 \mathrm{mg} / \mathrm{mL}$. Calibration standard solutions were prepared by serial dilution of primary stock solution with $50 \%$ methanol-water to obtain concentrations of $0.05,0.1,0.5,1,5$ and $10 \mu \mathrm{g} / \mathrm{mL}$, respectively. Quality control (QC) samples were prepared at $0.2 \mu \mathrm{g} / \mathrm{mL}, 2 \mu \mathrm{g} / \mathrm{mL}$ and $6 \mu \mathrm{g} / \mathrm{mL}$ of low, medium and high concentration levels, which were used in developed analytical methods. All solutions were kept at $4{ }^{\circ} \mathrm{C}$ in fridge and were brought to room temperature prior to use. The dried tubers of G. elata were finely powered, and $0.5 \mathrm{~g}$ of the powder was precisely weighed and extracted with $50 \%$ methanol-water $(1: 20, w / v)$ for $1 \mathrm{~h}$ by ultrasound. After extraction, the mixture was centrifuged at $3000 \mathrm{rpm}$ for $10 \mathrm{~min}$. The supernatant was collected and filtered by $0.22 \mu \mathrm{m}$ filter membrane. $5 \mu \mathrm{L}$ of filtrate was dilute with $50 \%$ methanol-water to $1 \mathrm{~mL}, \mathrm{~A} 10 \mu \mathrm{L}$ aliquot was injected for HPLC-FLD analysis. 


\subsection{Hydrolysis}

\subsubsection{Base Hydrolysis}

G. elata extract $(50 \mu \mathrm{L})$ was added with $1 \mathrm{~mL}$ of $0.5 \mathrm{~mol} / \mathrm{L}$ sodium hydroxide solution. Sample solutions were maintained at $80^{\circ} \mathrm{C}$ for $0.5 \mathrm{~h}$ in a water bath. After hydrolysis, the sample was neutralized with $0.5 \mathrm{~mol} / \mathrm{L}$ hydrochloric acid solution, and diluted with $50 \%$ methanol-water to $2 \mathrm{~mL}$. $200 \mu \mathrm{L}$ of sample was taken and added $800 \mu \mathrm{L}$ of $50 \%$ methanol-water. A $2 \mu \mathrm{L}$ aliquot was injected for HPLC-FLD analysis.

\subsubsection{Base-Enzymatic Hydrolysis}

Sodium acetate $(5.4 \mathrm{~g})$ was dissolved in water $(50 \mathrm{~mL})$, and the $\mathrm{pH}$ adjusted to 4.60 with acetic acid in a $100 \mathrm{~mL}$ volumetric flask to prepare sodium acetate buffer solution. $\beta$-D-Glucosidase was dissolved in sodium acetate buffer solution. Fifty $\mu \mathrm{L}$ of G. elata extract was added with $50 \mu \mathrm{L}$ of $0.5 \mathrm{~mol} / \mathrm{L}$ sodium hydroxide solution. Sample solutions were maintained at $80^{\circ} \mathrm{C}$ for $0.5 \mathrm{~h}$ in a water bath. After hydrolysis, the sample was neutralized with $0.5 \mathrm{~mol} / \mathrm{L}$ hydrochloric acid solution, then added $500 \mu \mathrm{L}$ of $4 \mathrm{mg} / \mathrm{mL}$ of $\beta$-D-glucosidase solution, the final volume was $1 \mathrm{~mL}$. Sample solutions were incubated for $3 \mathrm{~h}$ in a vortex mixer at $1000 \mathrm{rpm}$. After incubation, the sample was heated at $85{ }^{\circ} \mathrm{C}$ for $4 \mathrm{~min}$ in a water bath, and $3 \mathrm{~mL}$ of ice-cold methanol was added to precipitate the proteins and vortex-mixed for $5 \mathrm{~min}$ when cooled. Then the samples were centrifuged at 12,000 rpm for $10 \mathrm{~min}$ at $4{ }^{\circ} \mathrm{C}$. The supernatant was transferred and filtered by $0.22 \mu \mathrm{m}$ filter membrane. $400 \mu \mathrm{L}$ of filtrate was taken and added with $600 \mu \mathrm{L}$ of $50 \%$ methanol-water solution for analysis. A $2 \mu \mathrm{L}$ aliquot was injected for HPLC-FLD analysis.

\subsection{Analytical Method Validation}

\subsubsection{Linearity and LOQ}

Calibration curves was plotted from standard samples in the concentration range of $0.05-10 \mu \mathrm{g} / \mathrm{mL}$ by plotting the peak area $(y)$ versus concentration $(x)$ of each analyte. The LOQ were defined as the concentration of lowest concentration standard in the calibration curve that was analyzed with a precision (RSD \%) not exceeding $20 \%$ and with a trueness between $80 \%$ and $120 \%$.

\subsubsection{Precision and Trueness}

The precision and trueness were determined by analyzing QC samples for three concentration levels. Each level was analyzed five replicates within 1 day for intra-day precision and trueness, while once a day during five consecutive days for inter-day precision and trueness. Precision was expressed as relative standard deviation (RSD), and trueness was calculated by relative error (RE) at each concentration level. For trueness and precision, the criterion for the acceptability of data should not deviate by $\pm 15 \%$ from the nominal concentration.

\subsubsection{Recovery of Extraction and Matrix Effect}

The GAS, HBA, PE, PB, PC and PA were added into the power sample and the extract of G. elata for determination of recovery of extraction and matrix effect, respectively. The spiked concentration of GAS, HBA, PE, PB, PC and PA was $0.5 \mu \mathrm{g} / \mathrm{mL}$, three duplicates of the mixed samples were further analyzed, respectively. The extraction recovery was determined by comparing the calculating concentration following extraction and HPLC assays with the spiked concentration. The matrix effect was calculated by comparing the different value of peak area between determined and origin value of extract with that of $50 \%$ methanol-water solution. 


\section{Conclusions}

At the present study, two novel strategies for quality control of G. elata were proposed and successfully applied to evaluate the quality of 47 batches of $G$. elata from eight localities. A comparison of the proposed strategies with the Pharmacopoeia and multiple markers methods was also investigated. The results showed that the novel TGH-B and TH-BE strategies, which determined the total GAS and HBA, and total HBA, could comprehensively reflect the content of total active ingredients in G. elata, providing an effective strategy for the quality control of G. elata, and suggesting the revision of the latest Chinese or European pharmacopoeias for the modernization of G. elata use.

Supplementary Materials: Supplementary materials are available online, Table S1: The content of six active ingredients in all batches of extract of G. elata, Table S2: Orthogonal enzymatic hydrolysis experiments, Table S3: Eight localities and quantity of G. elata.

Acknowledgments: This work is financially supported by the National Natural Science Foundation of China (No. 81603266), Ningbo Natural Science Foundation (No. 2017A610258). In addition, the work was also sponsored by a K.C. Wong Magna Fund in Ningbo University and Ningbo University Talent Project. Jinyan Xu in the Affiliated Hospital of Medical School of Ningbo University provided some suggestions and modified the language in the paper.

Author Contributions: Chunlan Tang conceived and designed the paper; Bingchu Wu, Zheng Zhang and Bocheng Yu performed the experiments and collected literature. Chunlan Tang and Jinyi Wu analyzed the data and wrote the paper.

Conflicts of Interest: The authors declare no conflict of interest. The founding sponsors had no role in the design of the study; in the collection, analyses, or interpretation of data; in the writing of the manuscript, and in the decision to publish the results.

\section{References}

1. Huang, G.B.; Zhao, T.; Muna, S.S.; Jin, H.M.; Park, J.I.; Jo, K.S.; Lee, B.H.; Chae, S.W.; Kim, S.Y.; Park, S.H.; et al. Therapeutic potential of Gastrodia elata Blume for the treatment of Alzheimer's disease. Neural Regen. Res. 2013, 8, 1061-1070. [CrossRef] [PubMed]

2. Teong, I.W.J.; Ko, A.A.R.; Li, M.; Heese, K.; Liang, W. Gastrodia elata decreases isoprenaline potency and enhances spontaneous phasic activity in the rat detrusor. Int. J. Physiol. Pathophysiol. Pharmacol. 2011, 3, 29-37. [PubMed]

3. Manavalan, A.; Ramachandran, U.; Sundaramurthi, H.; Mishra, M.; Sze, S.K.; Hu, J.-M.; Feng, Z.W.; Heese, K. Gastrodia elata Blume (tianma) mobilizes neuro-protective capacities. Int. J. Biochem. Mol. Biol. 2012, 3, 219-241. [PubMed]

4. Ahn, E.K.; Jeon, H.J.; Lim, E.J.; Jung, H.J.; Park, E.H. Anti-inflammatory and anti-angiogenic activities of Gastrodia elata Blume. J. Ethnopharmacol. 2007, 110, 476-482. [CrossRef] [PubMed]

5. Hsieh, C.L.; Chiang, S.Y.; Cheng, K.S.; Lin, Y.H.; Tang, N.Y.; Lee, C.J.; Pon, C.Z.; Hsieh, C.T. Anticonvulsive and free radical scavenging activities of Gastrodia elata Bl. in kainic acid-treated rats. Am. J. Chin. Med. 2001, 29, 331-341. [CrossRef] [PubMed]

6. Hsieh, C.L.; Tang, N.Y.; Chiang, S.Y.; Hsieh, C.T.; Lin, J.G. Anticonvulsive and free radical scavenging actions of two herbs, Uncaria rhynchophylla (MIQ) Jack and Gastrodia elata BL., in kainic acid-treated rats. Life Sci. 1999, 65, 2071-2082. [CrossRef]

7. Kumar, H.; Kim, I.S.; More, S.V.; Kim, B.W.; Bahk, Y.Y.; Choi, D.K. Gastrodin Protects Apoptotic Dopaminergic Neurons in a Toxin-Induced Parkinson's Disease Model. Evid. Based Complement. Altern. Med. 2013, 2013, 514095. [CrossRef] [PubMed]

8. Chen, S.; Hao, X.W.; Yu, L.; Zhang, P.; Cao, W.; Chen, H.Y.; Zhu, D.L. Gastrodin causes vasodilation by activating KATP channels in vascular smooth muscles via PKA-dependent signaling pathway. J. Recept. Signal Transduct. Res. 2017, 37, 543-549. [CrossRef] [PubMed]

9. Zhan, H.D.; Zhou, H.Y.; Sui, Y.P.; Du, X.L.; Wang, W.; Dai, L.; Sui, F.; Huo, H.R.; Jiang, T.L. The rhizome of Gastrodia elata Blume-An ethnopharmacological review. J. Ethnopharmacol. 2016, 189, 361-385. [CrossRef] [PubMed] 
10. Chen, W.C.; Lai, Y.S.; Lu, K.H.; Lin, S.H.; Liao, L.Y.; Ho, C.T.; Sheen, L.Y. Method development and validation for the high-performance liquid chromatography assay of gastrodin in water extracts from different sources of Gastrodia elata Blume. J. Food Drug Anal. 2015, 23, 803-810. [CrossRef] [PubMed]

11. Shu, G.W.; Yang, T.M.; Wang, C.Y.; Su, H.W.; Xiang, M.X. Gastrodin stimulates anticancer immune response and represses transplanted H22 hepatic ascitic tumor cell growth: Involvement of NF-kappa B signaling activation in CD4+T cells. Toxicol. Appl. Pharm. 2013, 269, 270-279. [CrossRef] [PubMed]

12. Tang, C.L.; Wang, L.; Liu, X.X.; Cheng, M.C.; Qu, Y.; Xiao, H.B. Comparative pharmacokinetics of gastrodin in rats after intragastric administration of free gastrodin, parishin and Gastrodia elata extract. J. Ethnopharmacol. 2015, 176, 49-54. [CrossRef] [PubMed]

13. Tang, C.L.; Wang, L.; Liu, X.X.; Cheng, M.C.; Xiao, H.B. Pharmacokinetic study of Gastrodia elata in rats. Anal. Bioanal. Chem. 2015, 407, 8903-8910. [CrossRef] [PubMed]

14. Cheng, J.T. Review: Drug therapy in Chinese traditional medicine. J. Clin. Pharmacol. 2000, 40, 445-450. [CrossRef] [PubMed]

15. Wang, L.; Xiao, H.B.; Liang, X.M.; Wei, L.X. Identification of phenolics and nucleoside derivatives in Gastrodia elata by HPLC-UV-MS. J. Sep. Sci. 2007, 30, 1488-1495. [CrossRef] [PubMed]

16. Ku, Y.R.; Liu, Y.C.; Hau, J.P.; Wen, K.C.; Lin, J.H.; Huang, W.F. Determination of parishin, parishins B and C in Gastrodiae rhizoma by HPLC. J. Food Drug Anal. 1995, 3, 287-293.

17. Zhao, Y.K.; Cao, Q.E.; Xiang, Y.Q.; Hu, Z.D. Identification and determination of active components in Gastrodia elata BL. by capillary electrophoresis. J. Chromatogr. A 1999, 849, 277-283. [CrossRef]

18. Zhang, Y.; Li, M.; Kang, R.-X.; Shi, J.-G.; Liu, G.-T.; Zhang, J.-J. NHBA isolated from Gastrodia elata exerts sedative and hypnotic effects in sodium pentobarbital-treated mice. Pharmacol. Biochem. Behav. 2012, 102, 450-457. [CrossRef] [PubMed]

19. Laschke, M.W.; van Oijen, A.E.V.; Koerbel, C.; Scheuer, C.; Menger, M.D. 4-Hydroxybenzyl alcohol: A novel inhibitor of tumor angiogenesis and growth. Life Sci. 2013, 93, 44-50. [CrossRef] [PubMed]

20. Huang, N.K.; Chern, Y.J.; Fang, J.M.; Lin, C.I.; Chen, W.P.; Lin, Y.L. Neuroprotective principles from Gastrodia elata. J. Nat. Prod. 2007, 70, 571-574. [CrossRef] [PubMed]

21. Shin, E.J.; Whang, W.K.; Kim, S.; Bach, J.H.; Kim, J.M.; Nguyen, X.K.T.; Nguyen, T.T.L.; Jung, B.D.; Yamada, K.; Nabeshima, T.; et al. Parishin C Attenuates Phencyclidine-Induced Schizophrenia-Like Psychosis in Mice: Involvements of 5-HT1A Receptor. J. Pharmacol. Sci. 2010, 113, 404-408. [CrossRef] [PubMed]

22. Yu, S.S.; Zhao, J.; Zhen, W.P.; Zhao, Y. Neuroprotective effect of 4-hydroxybenzyl alcohol against transient focal cerebral ischemia via anti-apoptosis in rats. Brain Res. 2010, 1308, 167-175. [CrossRef] [PubMed]

23. Kim, I.S.; Choi, D.-K.; Jung, H.J. Neuroprotective Effects of Vanillyl Alcohol in Gastrodia elata Blume Through Suppression of Oxidative Stress and Anti-Apoptotic Activity in Toxin-Induced Dopaminergic MN9D Cells. Molecules 2011, 16, 5349-5361. [CrossRef] [PubMed]

24. Li, M.F.; Du, Y.; Wang, L.; Jiang, L.L.; Ma, X.D.; Zhou, P.; Li, P.; Li, H.J. Efficient Discovery of Quality Control Markers for Gastrodia elata Tuber by Fingerprint-Efficacy Relationship Modelling. Phytochem. Anal. 2017, 28, 351-359. [CrossRef] [PubMed]

25. Wang, L. Studies on Chemical Constituents and Quality Control of Gastrodia elata; Dalian Institute of Chemical Physics, Chinese Academy of Sciences: Dalian, China, 2007.

26. Ma, X.D.; Fan, Y.X.; Jin, C.C.; Wang, F.; Xin, G.Z.; Li, P.; Li, H.J. Specific targeted quantification combined with non-targeted metabolite profiling for quality evaluation of Gastrodia elata tubers from different geographical origins and cultivars. J. Chromatogr. A 2016, 1450, 53-63. [CrossRef] [PubMed]

27. Tao, J.; Qian, C.; Tang, Z.; Chen, P.; Wang, Y.; Han, Y. Chemical fingerprint technique and its application in the classification and quality assessment of the Gastrodia tuber. Afr. J. Biotechnol. 2011, 10, 16746-16756. [CrossRef]

28. Zhong, R.X.; Feng, G.F.; Liu, Y.J.; Zhong, L.; Wu, C.J.; Wan, J. Origin discrimination and quality evaluation of Gastrodiae rhizoma (Orchidaceae) by high-performance liquid chromatographic fingerprint. J. Pharm. Res. 2016, 15, 1535. [CrossRef]

29. Zengin, G.; Menghini, L.; Malatesta, L.; De Luca, E.; Bellagamba, G.; Uysal, S.; Aktumsek, A.; Locatelli, M. Comparative study of biological activities and multicomponent pattern of two wild Turkish species: Asphodeline anatolica and Potentilla speciosa. J. Enzyme Inhib. Med. Chem. 2016, 31, 203-208. [CrossRef] [PubMed] 
30. Chinese Pharmacopoeia Commission. Pharmacopoeia of the People's Republic of China; Chinese Medical Science and Technology Press: Beijing, China, 2015; pp. 58-59.

31. European Directorate for the Quality of Medicines and Healthcare (EDQM). European Pharmacopoeia, 8th ed.; EDQM: Strasbourg, France, 2014; pp. 128-132.

32. Tang, C.L.; Wang, L.; Cheng, M.C.; Zhang, X.Z.; Liu, X.Y.; Xiao, H.B. Rapid and sensitive analysis of parishin and its metabolites in rat plasma using ultra high performance liquid chromatography-fluorescence detection. J. Chromatogr. B 2014, 973, 104-109. [CrossRef] [PubMed]

33. Tang, C.L.; Wang, L.; Li, J.J.; Liu, X.X.; Cheng, M.C.; Xiao, H.B. Analysis of the metabolic profile of parishin by ultra-performance liquid chromatography/quadrupole-time of flight mass spectrometry. Biomed. Chromatogr. 2015, 29, 1913-1920. [CrossRef] [PubMed]

34. Li, Z.; Wang, Y.; Ouyang, H.; Lu, Y.; Qiu, Y.; Feng, Y.; Jiang, H.; Zhou, X.; Yang, S. A novel dereplication strategy for the identification of two new trace compounds in the extract of Gastrodia elata using UHPLC/Q-TOF-MS/MS. J. Chromatogr. B 2015, 988, 45-52. [CrossRef] [PubMed]

35. Lu, G.W.; Zou, Y.J.; Mo, Q.Z. Kinetic aspects of absorption, distribution, metabolism and excretion of 3H-gastrodin in rats. Acta Pharm. Sinica 1985, 20, 167-172.

36. Lin, L.C.; Chen, Y.F.; Lee, W.C.; Wu, Y.T.; Tsai, T.H. Pharmacokinetics of gastrodin and its metabolite p-hydroxybenzyl alcohol in rat blood, brain and bile by microdialysis coupled to LC-MS/MS. J. Pharm. Biomed. Anal. 2008, 48, 909-917. [CrossRef] [PubMed]

37. Wang, Q.; Chen, G.S.; Zeng, S. Distribution and metabolism of gastrodin in rat brain. J. Pharm. Biomed. Anal. 2008, 46, 399-404. [CrossRef] [PubMed]

38. An, S.J.; Park, S.K.; Hwang, I.K.; Choi, S.Y.; Kim, S.K.; Kwon, O.S.; Jung, S.J.; Baek, N.I.; Lee, H.Y.; Won, M.H.; et al. Gastrodin decreases immunoreactivities of gamma-aminobutyric acid shunt enzymes in the hippocampus of seizure-sensitive gerbils. J. Neurosci. Res. 2003, 71, 534-543. [CrossRef] [PubMed]

39. Li, H.B.; Chen, F. Preparative isolation and purification of gastrodin from the Chinese medicinal plant Gastrodia elata by high-speed counter-current chromatography. J. Chromatogr. A 2004, 1052, 229-232. [CrossRef] [PubMed]

40. Lin, L.C.; Chen, Y.F.; Tsai, T.R.; Tsai, T.H. Analysis of brain distribution and biliary excretion of a nutrient supplement, gastrodin, in rat. Anal. Chim. Acta 2007, 590, 173-179. [CrossRef] [PubMed]

Sample Availability: Samples of all extracts are available from the authors.

(C) 2018 by the authors. Licensee MDPI, Basel, Switzerland. This article is an open access article distributed under the terms and conditions of the Creative Commons Attribution (CC BY) license (http://creativecommons.org/licenses/by/4.0/). 